\title{
A STUDY ON JOB POSTURES AND MUSCULOSKELETAL ILLNESSES IN DENTISTS
}

\section{EHSAN RAFEEMANESH ${ }^{1}$, ZAHRA JAFARI ${ }^{2}$, FARZAD OMIDI KASHANI', and FARZANEH RAHIMPOUR ${ }^{1}$}

\author{
${ }^{1}$ Mashhad University of Medical Sciences, Mashhad, Iran \\ Occupational Medicine, Faculty of Medicine \\ ${ }^{2}$ Mashhad University of Medical Sciences, Mashhad, Iran \\ Occupational \& Environmental Health Department, Torghabe-Shandiz Health Center \\ ${ }^{3}$ Mashhad University of Medical Sciences, Mashhad, Iran \\ Orthopedic, Faculty of Medicine
}

\begin{abstract}
Objectives: Musculoskeletal disorders (MSDs) compose a large part of occupational diseases in dental professionals, prevention of which is dependent on assessment and improvement of job postures by means of ergonomic interventions. This study was aimed at evaluation of ergonomic conditions of the profession of dentists and also at assessing the relationship between MSDs and conditions of work. Materials and Methods: This cross-sectional study was performed among 65 dentists using the method of Rapid Entire Body Assessment (REBA). The prevalence of MSDs was obtained by the use of the Nordic Musculoskeletal Questionnaire (NMQ). Results: In this investigation, the prevalence of MSDs for different body parts was: $75.9 \%$ for the neck, $58.6 \%$ for the shoulders, $56.9 \%$ for the upper back, $48.3 \%$ for the lower back and $44.8 \%$ for the wrist. Job analysis by the use of REBA showed that $89.6 \%$ of limbs in group A and $79.3 \%$ of limbs in group B had a score $>4$. Only neck and lower back pain have significant relationship with the risk levels obtained using the REBA method. Conclusions: It can be concluded that work postures of dentists need to be improved. In addition to education, work station design, rest period during work and regular physical activities should be taken into account.
\end{abstract}

Key words:

Musculoskeletal disorders, Dentists, REBA

\section{INTRODUCTION}

Work-related Musculoskeletal Disorders (WMSDs) are any kind of disorders in the functioning of bones, muscles, tendons, and joints which are usually preceded by long term work involving performance of repetitive activities. Working in awkward positions, doing repetitive tasks, carrying heavy loads repeatedly, lack of rest period during the work shift as well as using non ergonomic chairs and unavailability of appropriate tools are the most important reasons for WMSDs among employees. Individual factors such as obesity, pregnancy and smoking are also effective in the development of such disorders [1]. Basing on the national study on burden of the diseases in Iran in 2004, back pain, knee arthritis, and other WMSDs were identified as the most important diseases of the country right after cardiovascular disorders [2].

This study was supported by the Vice Chancellor for Research of Mashhad University of Medical Sciences with registration number 88413.

Received: December 21, 2012. Accepted: July 23, 2013.

Corresponding author: E. Rafeemanesh, Occupational Medicine Department, Faculty of Medicine, Pardis, Park square, Mashhad, Iran (e-mail: rafeemaneshe@mums.ac.ir). 
Musculoskeletal disorders in working population lead to the increased disability, productivity loss, treatment costs, time loss and early disability and thus, have a number of negative outcomes in different fields of social, economical and health aspects for human communities. In the United States, about one million people are absent from work annually because of the treatment and rehabilitation of these disorders and the cost of compensation is estimated at 13-20 billion dollars [3]. In dentistry, due to the repetitive activities, long term working in static postures, inappropriate working conditions as well as usage of inappropriate tools musculoskeletal disorders are likely to occur - the prevalence of these disorders in different global studies is reported to occur in back, neck, shoulders and arms from $64 \%$ to $93 \%$ of the cases [4]. In Iran, on the basis of the study carried out in Birjand, the prevalence of neck pain was found to constitute $65 \%$ of the ailments among dentists, back pain - 60\%, and shoulder pain $-38 \%$ [5]. The present study was aimed to determine the prevalence of musculoskeletal disorders and job postures analysis in dentists and the possible relationship between MSDs and occupational and personal factors in this job.

\section{MATERIALS AND METHODS}

This study was carried out among 65 dentists working in the Health Centers of Mashhad. The study was granted ethical approval by the Ethical Committee of Mashhad University of Medical Sciences (MUMS). For evaluation of the musculoskeletal complaints the Standardized Nordic questionnaire was applied. In order to score job postures the Rapid Entire Body Analysis (REBA) was used. Additionally, the dentists were observed in their workplace. The Nordic questionnaire was planned by Kuorinka in the Scandinavian Professional Health Institute in order to determine the epidemic of musculoskeletal disorders. It includes 4 parts of general questions, limb musculoskeletal complaints (neck, shoulders, back, wrist, knee, and ankle), work absenteeism and working restrictions resulting from these disorders [6].

The Nordic questionnaire was filled in by 58 dentists performing tasks such as restoration and teeth extraction. Dentists having less than 2 years of job experience or musculoskeletal disorders for reasons other than work, such as congenital and collagen vascular diseases, were excluded from the study. In order to study working postures, the REBA method was used. This method was designed by Hignettz Mc Alamney in 1998 for the purpose of analysis of job postures [7]. In REBA, the limbs are divided into 2 groups:

- A - neck, upper and lower back, and legs;

- B - shoulders, elbows, and wrists.

By observing each job posture neck, body, upper and lower limbs are scored on the basis of their posture angles. Basing on the individual scores, a total score is achieved and the need for correction of that posture is identified (based on Table 1). In our study job postures were evaluated by filming the dentists for $30 \mathrm{~min}$ and also by observing their job posture directly for the sake of evaluation and recording it in REBA scoring worksheet. The scores of different limbs were achieved and finally basing on the final score and standard definitions, the level of actions was determined. Data was analyzed by the use of SPSS version 11.5 using the independent sample t-test, $\mathrm{Chi}^{2}$ and Fisher exact tests.

Table 1. REBA action levels

\begin{tabular}{lcll}
\hline $\begin{array}{c}\text { Action } \\
\text { level }\end{array}$ & $\begin{array}{c}\text { REBA } \\
\text { score }\end{array}$ & Risk level & $\begin{array}{c}\text { Action } \\
\text { (including further assessment) }\end{array}$ \\
\hline 0 & 1 & negligible & not necessary \\
1 & $2-3$ & low & may be necessary \\
2 & $4-7$ & medium & necessary \\
3 & $8-10$ & high & soon necessary \\
4 & $11-15$ & very high & necessary now \\
\hline
\end{tabular}




\section{RESULTS}

All the dentists working in the Health Centers of Mashhad (65 people) were invited to participate in the study. 60 dentists filled in the Nordic questionnaire. 2 of them were excluded from the study because of chronic rheumatologic diseases they suffered from. Out of 58 studied dentists, 49 were males $(84.5 \%)$ and 9 were females (15.5\%). Personal information concerning the participants is presented in Table 2. Basing on the Body Mass Index (BMI), one of the participants $(1.7 \%)$ was skinny, 27 people (46.7\%) were of normal weight, 19 people $(32.7 \%)$ were overweight and 11 people $(18.9 \%)$ were fat.

Table 2. Demographic information concerning the participants

\begin{tabular}{lc}
\hline \multicolumn{1}{c}{ Variables } & $\begin{array}{c}\text { Participants } \\
(\mathrm{N}=58)\end{array}$ \\
\hline Age (year), M $\pm \mathrm{SD}$ & $43.2 \pm 3.2$ \\
Height $(\mathrm{cm}), \mathrm{M} \pm \mathrm{SD}$ & $171.1 \pm 5.8$ \\
Weight $(\mathrm{kg}), \mathrm{M} \pm \mathrm{SD}$ & $78.2 \pm 14.1$ \\
BMI, M $\pm \mathrm{SD}$ & $26.7 \pm 4.2$ \\
Employment duration (year), $\mathrm{M} \pm \mathrm{SD}$ & $17.6 \pm 4.8$ \\
Smoking [n $(\%)]$ & $6(0.1)$ \\
\hline
\end{tabular}

$\mathrm{M}$ - mean; SD - standard deviation.

Figure 1 shows the prevalence of complaints concerning different body parts. The prevalence of disorders among females occurred in shoulders $-88.9 \%$, neck $-66.7 \%$, upper back $-55.6 \%$, lower back $-44.4 \%$, knee $-44.4 \%$, and wrist $-33.3 \%$, respectively. The prevalence of disorders among males concerned neck - 77.6\%, upper back $57.1 \%$, shoulders $-53.1 \%$, lower back - $49 \%$, wrist $46.9 \%$, and knee $-44.9 \%$, respectively.

The prevalence of disorders in the A group (neck, lower and upper back, and legs) was $87.9 \%$, which was higher than in the B group (shoulders, arms and wrists) in which it amounted to $75.9 \%$.

In the Health Centers, dentists usually work 6 hours per day. Daily, about 15 patients refer to these clinics:

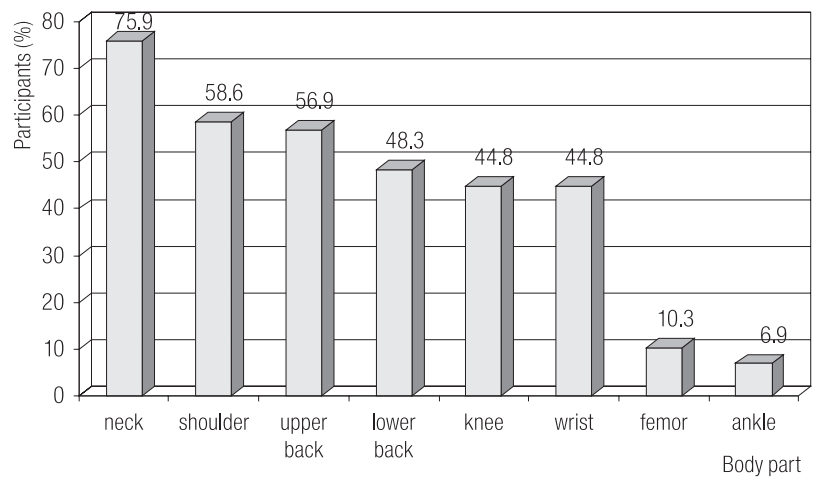

Fig. 1. Prevalence of musculoskeletal complaints in the study participants $(\mathrm{N}=58)$ in the past 12 months

6 patients for extraction, 4 patients for restoration and $5 \mathrm{pa}-$ tients for physical examination. Extraction and physical examinations are usually performed in standing positions while restoration is performed in a sitting position. Only few of these governmental clinics have dental assistance. According to our observations and standard tables, the scores of $\mathrm{A}$ and $\mathrm{B}$ groups in different job postures include restoration and upper and lower jaw teeth extraction and were studied by the use of REBA checklist. The mean REBA scores in extraction activities were as follows: for the neck -3.2 (more than $20^{\circ}$ flexion or twisting/side bending), for the trunk - 3.6 (flexion 20-60 $)$ and for the shoulder -3.9 (flexion: $45-90^{\circ}$ ). The mean REBA scores in restoration activities were as follows: for the neck - 3.1 (more than $20^{\circ}$ flexion), for the trunk -2.3 (flexion: $0-20^{\circ}$ ) and for the shoulder -2.7 (flexion: $20-45^{\circ}$ ).

The highest score of the A group was 8 and the lowest was 3 . The highest score of the B group was 7, and the lowest was 3. Basing on the scores of A and B groups, the score of applied force and muscle and the final score using REBA standard table, the action level was determined. The highest score of REBA was 11 and the lowest was 4. The data analysis of job postures of the study group is presented in Table 3. The final results of job postures analysis by the use of REBA showed that extraction of the right lower jaw teeth (with the score of 11), extraction of the left lower jaw teeth (with the score of 10) and extraction of the right upper jaw 
Table 3. The results of dentists' job posture analysis based on REBA

\begin{tabular}{llccc}
\hline \multirow{2}{*}{ Rows } & \multicolumn{1}{c}{ Job posture } & \multicolumn{2}{c}{ Limbs score } & Final REBA \\
\cline { 3 - 4 } & & group A & group B & score \\
\hline 1 & restoration of the right lower jaw teeth & 5 & 3 & 5 \\
2 & restoration of the upper jaw & 5 & 7 & 9 \\
3 & restoration of the right upper jaw teeth & 5 & 4 & 6 \\
4 & restoration of the left lower jaw teeth & 5 & 3 & 5 \\
5 & restoration of the right anterior teeth of lower and upper jaw & 3 & 4 & 4 \\
6 & restoration of the right upper and lower jaw anterior teeth & 5 & 5 & 7 \\
7 & restoration of the left upper jaw teeth & 6 & 5 & 9 \\
8 & extraction of the right upper jaw teeth & 6 & 5 & 9 \\
9 & extraction of the left lower jaw teeth & 7 & 7 & 10 \\
10 & extraction of the right lower jaw teeth & 8 & 7 & 11 \\
\hline
\end{tabular}

teeth (with the score of 9), as well as restoration of the upper jaw teeth (with the score of 9) had the worst job postures. Totally, in this study, on the basis of the results obtained using the REBA method, in the A group, 8.6\% were with high risk, $81 \%$ with average risk, and $8.3 \%$ with low risk. Dentists extracting the right lower jaw teeth had the score corresponding to a very high risk (11). Therefore, in their case posture improvement requires immediate action.

T-test and $\mathrm{Chi}^{2}$ test were used to determine the effect of personal factors such as sex, age, height, weight as well as job history on the prevalence of symptoms in different limbs. The results showed that age had a significant effect on the prevalence of symptoms in the back, upper back and knees - the average age in the symptomatic group with regard to these parts was higher than in the asymptomatic group $(p<0.05)$. Among complaints concerning different limbs in the study group a statistically significant relationship was observed only between shoulder pain and $\operatorname{sex}(p<0.05)$. This disorder in female dentists was more common than in the case of males. In this study, the relationship between BMI and upper back $(p=0.07)$ and lower back $(p=0.03)$ disorders was significant. No statistically significant relationship between musculoskeletal disorders and job history was observed. Similarly, no significant relationships were reported between other disorders and BMI, age, and job history.

Table 4 displays the relationship between musculoskeletal complaints in different parts of the body and the risk level of REBA analyzed by $\mathrm{Chi}^{2}$ and exact tests.

Table 4. The relationship between musculoskeletal complaints in different body parts and the REBA risk level within the studied population

\begin{tabular}{lcccc}
\hline \multirow{2}{*}{ Body part } & \multicolumn{3}{c}{ Risk level in REBA $(\mathrm{N}=58)$} & \multirow{2}{*}{$\mathrm{p}^{*}$} \\
\cline { 2 - 4 } & medium & high & very high & \\
\hline Neck & 50.0 & 43.2 & 6.8 & 0.037 \\
Shoulder & 55.9 & 35.3 & 8.8 & 0.050 \\
Upper back & 45.5 & 45.5 & 9.1 & 0.050 \\
Lower back & 60.7 & 35.7 & 3.6 & 0.041 \\
Knees & 65.4 & 26.9 & 7.7 & 0.050 \\
Wrist & 50.0 & 42.3 & 7.7 & 0.050 \\
Thighs & 83.3 & 16.7 & 0.0 & 0.050 \\
Ankle & 50.0 & 50.0 & 0.0 & 0.050 \\
\hline
\end{tabular}

${ }^{*}$ Chi $^{2}$ test.

$27.2 \%$ of the participants of this study have been absent from work in the past year because of some kind of musculoskeletal disorder (25.3\% for 1-7 days and 1.9\% 
for 8-30 days). The major reason for being absent from work in the past year was back pain $(7.1 \%)$ and the main reason for job restriction while doing the job was shoulder pain (37\%), upper back (31.6\%) and neck (31.4\%) disorders.

\section{DISCUSSION}

The most common musculoskeletal complaints among the dentists who participated in this study were related to neck, shoulders, upper back, lower back, and wrists respectively. The complaints related to the A group were more prevalent than the ones related to the $\mathrm{B}$ group. The prevalence of disorders among females concerned shoulders, neck, upper and lower back while among males: neck, upper back, shoulders, and lower back respectively. The prevalence of shoulder and ankle disorders in females was higher than in males; on the contrary neck, back and wrist complaints were more common in males. In this study back disorder was less prevalent compared to the other studies. Taking into account the significant relationship between BMI and lower and upper back disorders, it can be concluded that being overweight and not being fit can increase the prevalence of such disorders. A significant relationship was observed between age and upper and lower back and knees disorders which should be taken into consideration in preventive plans. The insignificant relationship between duration of the employment and musculoskeletal disorders, implies that all dentists, regardless of employment duration, are exposed to such diseases and therefore, preventive programs should be planned just at the beginning of the employment.

In the study carried out in Nepal in 2006, the WMSDs among dentists were studied. 68 dentists participated in the study by filling in a questionnaire. According to this study, back pain was the most prevalent disorder among the dentists and almost $80 \%$ of the participants complained about it. Neck pain was the next most prevalent complaint - in $58.8 \%$ and finally shoulders pain - in $47 \%$ of this group. Shoulder disorders in females were two times more prevalent than in males. The researchers of Nepal study finally concluded that musculoskeletal complaints are extremely prevalent among dentists and the only way to reduce them is doing regular exercise [8]. In our study, ten job postures which were most frequently observed were evaluated by the use of the REBA method. The A group was more at risk than the B group from the point of ergonomics which was concordant with a higher prevalence of complaints in the A group based on the Nordic questionnaire data. Most of the limbs in dentistry are at a higher than average risk therefore, improvement of this situation is necessary. The worst posture was related to extraction of the lower jaw teeth which can be a result of the unbalanced standing position and using a lot of force in this task. Restoration tasks, especially in the upper jaw, mostly cause awkward posture as they require more observation and attention. In dentistry, dynamic and static activities predispose dentists for musculoskeletal disorders but this study showed that only neck and lower back pain have significant relationship with the risk levels obtained by the use of the REBA method. This association, however, was not displayed in other parts of the body. The most important reasons for the increased REBA mean for the neck and lower back were: unfavourable position of the instrument table, lack of assistance and lack of proper ergonomic seat. In 2006 Lagott \& Smith assessed musculoskeletal disorders among Australian dentists. In their study a standard questionnaire was filled in by 285 dentists. $79.25 \%$ of them mentioned at least one musculoskeletal disorder in the past year. The most common musculoskeletal complaints included: 1 - neck pain (57.5\%), 2 - back pain (53.7\%), 3 shoulder pain (53.3\%). More than 1/3 of the dentists had been under treatment or medical care because of musculoskeletal disorders in the past year. About 10\% of the dentists had been absent from work in the past year (on average 11.5 days) [9]. In another study carried out by Marklin \& Cherni in 2005, the postures of dentists and dental hygienists were studied. The job postures of 10 dentists 
and 10 dental hygienists were studied by filming. $78 \%$ of dentists and $66 \%$ of dental hygienists were sitting while working. Dentists and dental hygienists were at neck flexion position for $50 \%$ and $85 \%$ of the working time respectively. Dentists were at shoulder abduction position for $50 \%$ of the working time. Totally, the postures of this study population were static [10].

Although in this study the most prevalent musculoskeletal complaints were related to neck and shoulder and these comprised the major cause of job restriction in dentists, the major reason for job absenteeism was back pain. According to the method of our study and its data collection, which was by means of a questionnaire, there might be a recall bias. Also, as the participants of our study were still working and the people who had left work because of musculoskeletal disorders were not evaluated, Healthy Worker Effect might be present. Therefore, the prevalence of musculoskeletal disorders might be larger than it seemed to be.

According to the results of our study, the following practical suggestions can be made in order to prevent musculoskeletal disorders in dentists working in the Health Centers:

1. Corrective measures in job postures by using ergonomic seats (with suitable arm rest and back support) and units so that there will be less need for neck and trunk bending or twisting.

2. Diminishing working hours and employing dental assistants to prevent pain and fatigue in different organs of the body.

3. Implementation of ergonomic training courses in order to increase the dentists' awareness.

4. Weight control and BMI balance as well as considering breaks during work.

\section{ACKNOWLEDGEMENTS}

The authors would like to thank Dr Baratali Arabnejad \& Dr Naser Khozeimeh for general support and Mohammad Javad Fahoul \& Dr Amin Samiei for their critical comments on this manuscript. The authors also wish to thank Dr. Ramin Sadeghi for revising the manuscript.

\section{REFERENCES}

1. Levy Barry S. Occupational and Environmental Health: Recognizing and Preventing Disease and Injury. 5th ed. Philadelphia: Lippincott Williams \& Wilkins; 2006. p. 488-516.

2. Naghavi M. [National study on burden of diseases in Iran]. Tehran: Ministry of Health and Medical Education; 2005. Persian.

3. Bernard BP. Musculoskeletal disorders and workplace factors A critical review of epidemiologic evidence for work-related musculoskeletal disorders of the neck, upper extremity, and low back [document on the Internet]. 1997 [cited 2012 Nov 2]. Available from URL: http://www.cdc.gov/niosh/docs/97-141.

4. Hayess M, Cockrell D, Smith DR. A systematic review of musculoskeletal disorders among dental professionals. Int J Dent Hyg. 2009;7(3):159-65, http://dx.doi.org/10.1111/ j.1601-5037.2009.00395.x.

5. Hoseini H. [Evaluation of ergonomic postures of dental professions by REBA in Birjand. Doctorate thesis]. Tehran: Tehran University of Medical Sciences; 2001. Persian.

6. Kuorinka I, Jonsson B, Kilbom A, Vinterberg H, BieringSorensen F, Andersson G, et al. Standardized Nordic questionnaires for the analysis of musculoskeletal symptoms. Appl Ergon. 1987;18:233-7, http://dx.doi.org/10.1016/00036870(87)90010-X.

7. Hignett S, McAtamney L. Rapid entire body assessment (REBA). Appl Ergon. 2000;31(2):201-5, http://dx.doi.org/10. 1016/S0003-6870(99)00039-3.

8. Shrestha BP, Singh GK, Niraula SR. Work related complaints among dentists. J Nepal Med Assoc. 2008;47(170):77-81.

9. Leggat PA, Smith DR. Musculoskeletal disorders self-reported by dentists in Queensland, Australia. Aust Dent J. 2006;51(4): 324-7, http://dx.doi.org/10. 1111/j.1834-7819.2006.tb00451.x.

10. Marklin RW, Cherney K. Working postures of dentists and dental hygienists. J Calif Dent Assoc. 2005;33(2):133-6.

This work is available in Open Access model and licensed under a Creative Commons Attribution-NonCommercial 3.0 Poland License - http://creativecommons.org/ licenses/by-nc/3.0/pl/deed.en. 